\title{
Effects of thunderstorms electric field on intensities of positrons, electrons and photons at Daocheng
}

\author{
Bing Zhao ${ }^{1}$, Daihui Huang ${ }^{1 *}$, Xunxiu Zhou ${ }^{1}, K_{\text {Kegu Axi }}{ }^{1}$, Xinhua Ma $^{2}$ \\ ${ }^{1}$ School of Physical Science and Technology,Southwest Jiaotong University, Chengdu \\ 610031,China \\ ${ }^{2}$ Institute of High Energy Physics, CAS,Beijing 100049, China \\ E-mail: hohamydsina.com
}

\begin{abstract}
Study on the intensity changes of the cosmic rays during thunderstorms is very important for understanding the acceleration mechanism of secondary charged particles caused by an atmospheric electric field. In this work, Monte Carlo simulations were performed with CORSIKA to study the intensity changes of positrons, electrons and photons in thunderstorms electric field. We found that the number of these secondary particles changes significantly. The variation amplitude is closely related to the strength and polarity of the electric field. A series fields were chosen in our simulations, ranging from $-1000 \mathrm{~V} / \mathrm{cm}$ to $1000 \mathrm{~V} / \mathrm{cm}$. The correlations of the intensity variations between positrons, electrons and photons were also discussed. The LHAASO (Large High Altitude Air Shower Observatory) project is located at Daocheng, Sichuan province, China, at an attitude of $4400 \mathrm{~m}$ above the sea level. As the main part of this project, the electromagnetic particle detector (ED) in the kilometer-square array (KM2A) and the water Cherenkov detector array (WCDA) are sensitive to the secondary positrons, electrons and photons in extensive air shower (EAS). Our simulation results may provide important information for giving reasonable explanations to the forthcoming experimental data of LHAASO.

Keywords: Thunderstorms electric field, Cosmic rays, Monte Carlo simulations, Secondary particles intensity
\end{abstract}

36th International Cosmic Ray Conference -ICRC2019-

July 24th - August 1st, 2019

Madison, WI, U.S.A.

\footnotetext{
* Speaker.
} 


\section{Introduction}

Observations indicate that the strength of electric fields generated inside thunderclouds can be up to $2 \mathrm{kV} / \mathrm{cm}[1,2]$. It was first presented by Wilson [3] that the secondary electron in cosmic rays might be accelerated to very high energy in strong electric fields. While the electron gains more energy from the electric field than it loses in various interactions with air, the energy of the electron will increase and lead to runaway. Gurevich et al. [4] put forward a new breakdown mechanism in 1992. Accelerated by the fields, the secondary electron may become runaway and ionize further molecules, which results in avalanche process. Marshall, Dwyer and Symbalisty et al. [5-7] built upon this theory, now commonly called a relativistic runaway electron avalanche (RREA).

Terrestrial gamma ray flash (TGF) was first discovered in 1994 by the Burst And Transient Source Experiment (BATSE) [8] and was thought to be related to thunderstorms. Since then, many scientists have devoted to looking for the high-energy electrons or the high-energy gamma rays radiated by bremsstrahlung. For years, multiple of terrestrial gamma flashes (TGFs) [9, 10] have been observed by satellite-borne experiments and masses of thunderstorm ground enhancements (TGEs) have been detected by ground-based experiments [11, 12]. It indicates that the RREA process is believed to be the reasonable explanations of these phenomena.

Several detection studies on correlations between the intensity of the cosmic ray and the electric field during thunderstorms were carried out at YBJ (4300 m a.s.l., Tibet, China). Wang et. al. [13] found that the count rates of muons did not always increase in the field, in some cases it would decline. Alexeenko et al. [14] found a decrease of muons intensity during thunderstorms. Bartoli et al. [15] observed the decrease phenomenon in the number of secondary electrons by ARGO-YBJ. These results are unable to be explained reasonable by the RREA mechanism. What are the correlations between the decreasing phenomena and thunderstorms electric field? Moreover, what is the acceleration mechanism for these abnormal results? In order to learn more about the acceleration mechanism and the intensity change, more theoretical, experimental and careful simulation results are needed.

The Large High Altitude Air Shower Observatory (LHAASO) located in Daocheng, Sichuan Province, China, is featured with high altitude ( 4400 m) and frequent thunderstorms in summer. It is beneficial to study the influence of atmospheric electric field on cosmic rays during thunderstorms, thereby providing information for understanding the physical acceleration mechanism of secondary charged particles in the electric field. As the main parts of this project, the electromagnetic particle detector (ED) in the kilometer-square array (KM2A) and the water Cherenkov detector array (WCDA) are sensitive to the secondary positrons, electrons and photons in extensive air shower (EAS). In this work, Monte Carlo simulations were performed with CORSIKA to study the effects of thunderstorm electric field on the secondary positrons, electrons and photons in cosmic rays at Daocheng. The simulations may provide important information for giving reasonable explanations to the forthcoming experimental data of LHAASO.

\section{Simulation setup}

CORSIKA is a detailed Monte Carlo program to study the evolution and properties of extensive air showers in the atmosphere [16]. The code of CORSIKA is 7.5700. QGSJETII-04 was used 
for the high-energy hadronic interactions while GHEISHA for the low energy ones. The primary shower includes proton and helium nuclei, with energy range from $14 \mathrm{GeV}$ to $1 \mathrm{PeV}$, corresponding to power law spectrum -2.7 and -2.64 , respectively. We assume primary shower with a zenith angle in the interval from $0^{\circ}$ to $70^{\circ}$. The values of the geomagnetic field components used in simulations are $B_{x}=34.6 \mathrm{uT}, B_{z}=35.7 \mathrm{uT}$, for the horizontal and vertical intensity, respectively. The shower number is $5.0 \times 10^{4}$.

It has been found that thunderclouds are distributed in the altitude range of 4-12 km. The strength of the electric field during thunderstorm can be high up to $1000 \mathrm{~V} / \mathrm{cm}$ or even higher, but mostly within $1000 \mathrm{~V} / \mathrm{cm}$. We applied vertical uniform electric field from $-1000 \mathrm{~V} / \mathrm{cm}$ to 1000 $\mathrm{V} / \mathrm{cm}$ in our simulations. It is lower than the threshold field strength, which results in a runaway breakdown process [6, 7]. The fields distribute from $6400 \mathrm{~m}$ to $4400 \mathrm{~m}$ above sea level (corresponding to the atmospheric depth $458-599 \mathrm{~g} / \mathrm{cm}^{2}$ ). Here, we defined the positive field was downward. In the positive field (pointed downward), the positrons (electrons) are accelerated (decelerated) downward; while in the negative field (pointed upward), the positrons (electrons) are decelerated (accelerated) downward. In view of the acceleration of the field, we set the energy cutoff at $0.1 \mathrm{MeV}$ for positrons, electrons and photons.

Note that the symbol $\gamma, e^{+}, e^{-}$and $e^{ \pm}$in this paper represent the photons, positrons, electrons and the sum of positrons and electrons, respectively.

\section{Simulation results}

Since electrons and positrons predominate in the secondary charged particles of the cosmic rays, and the apparent acceleration (or deceleration) of electric field on small quantity electrons (or positrons) is more obvious, the effects of electric field on electrons (or positrons) were properly taken into account in this work. And, though the electric field does not act on the photons directly, it still has a significant effect on them. So, our simulations were also concerned with the influence of electric field on photons.

\subsection{The longitudinal development of $e^{+}, e^{-}$and $\gamma$ in electric fields}

In order to understand more complete images of the longitudinal development of the secondary particles, we studied the case without adding any electric fields at first.

In Fig.1, the number of $e^{+}, e^{-}$and $\gamma$ is plotted as a function of atmospheric depth in absence of an electric field. The blue, purple and red lines represent the development of $e^{+}, e^{-}$and $\gamma$, respectively. As shown in the figure, the laws of the longitudinal development of $e^{+}, e^{-}$and $\gamma$ are in good agreement. However, the number of photons is huge. It is much larger than the total number of $e^{+}, e^{-}$. High up in the atmospheric depth, the ratio of $N_{\gamma} / N_{e}{ }^{ \pm}$increase gradually $\left(N_{\gamma}\right.$ and $N_{e}{ }^{ \pm}$represent the number of photons and the total number of positrons and electrons, respectively). When reaching the detection surface at Daocheng, it is up to $\sim 9.1$. So, in the secondary particles, photons cannot be ignored.

Fig. 2 and Fig. 3 show the simulation results in electric field strength of $1000 \mathrm{~V} / \mathrm{cm}$, the number of particles is plotted as a function of atmospheric depth. As shown in the figure 2, the number of positrons exceeds the number of electrons in the positive field (accelerating the positrons), causing a positive charge excess. At the detection surface of LHAASO site, the number increase of 


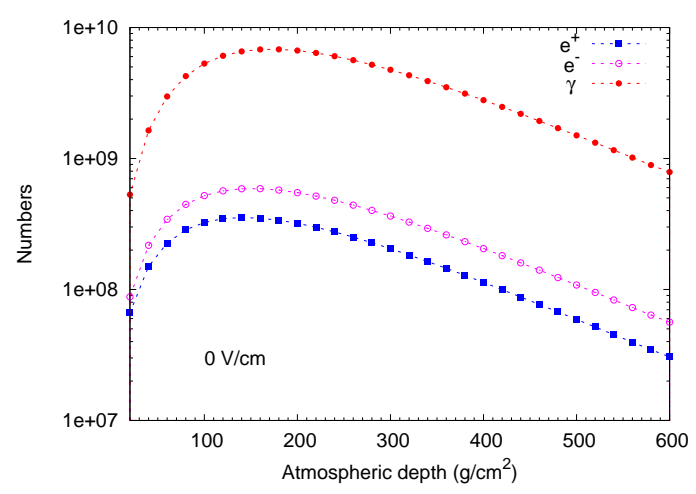

Fig. 1: Number of $e^{+}, e^{-}$and $\gamma$ as a function of atmospheric depth in absence of an electric field.

positrons is about $98.8 \%$, the ratio of electrons to positrons is $\sim 0.66$. In Fig. 3, an increase in the number of electrons occurs when the negative field is switched on. While the number increase of electrons is $\sim 133.7 \%$, the ratio of electrons to positrons is $\sim 6.30$. Obviously, the effect of field on electrons is larger than positrons. We can apparently see from Fig. 2 and Fig. 3, whether it is in a positive electric field or in a negative one, the number of photons is always increasing.

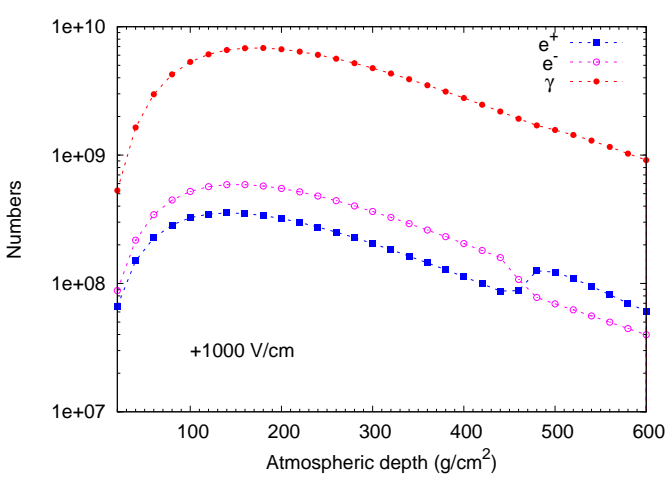

Fig. 2: Number of $e^{+}, e^{-}$and $\gamma$ as a function of atmospheric depth in $1000 \mathrm{~V} / \mathrm{cm}$.

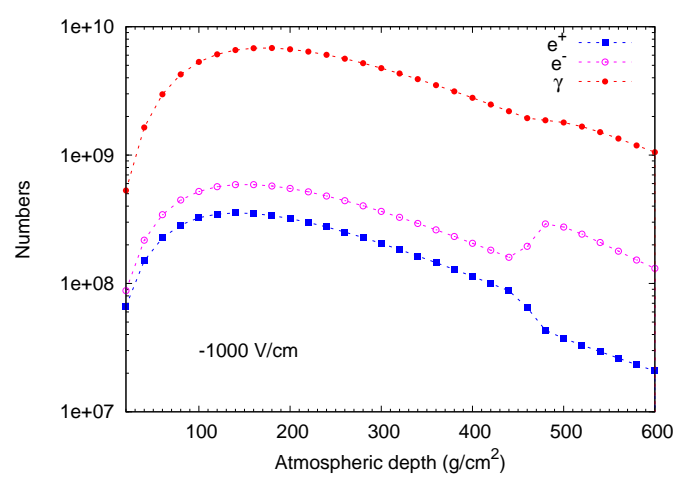

Fig. 3: Number of $e^{+}, e^{-}$and $\gamma$ as a function of atmospheric depth in $-1000 \mathrm{~V} / \mathrm{cm}$.

The percent changes of particle numbers in the thunderstorm electric field as a function of atmospheric depth are shown in Fig. 4 and Fig. 5. The number percentage change trends of $e^{ \pm}$and $\gamma$ with altitude are almost the same. As shown in Fig. 4, in an electric field of $1000 \mathrm{~V} / \mathrm{cm}$, when the atmospheric depth increases, both the number of $\gamma$ and the total number of $e^{+}$and $e^{-}$decrease at first, then increase rapidly, and then the rate of increase goes down. Reaching the detection surface at Daocheng, the percentage increase amplitude of $e^{ \pm}$and $\gamma$ are $15.8 \%$ and $15.9 \%$, respectively. However, the shape of the variation curve in Fig. 5 is quite different from that in Fig. 4. In -1000 $\mathrm{V} / \mathrm{cm}$, the total number of $e^{+}$and $e^{-}$keeps increasing, so does the number of $\gamma$. Corresponding to $e^{ \pm}$and $\gamma$, the percentage increases are up to $\sim 74.7 \%$ and $\sim 33.7 \%$.

By comparing the intensity variation amplitudes in different polarity field, we can draw a conclusion that the flux change of secondary particles in negative field is greater than that in positive one. It is closely related to the polarity of the field. Not only that, the variation amplitude also depends on the strength of the field, as the simulation results described below. 


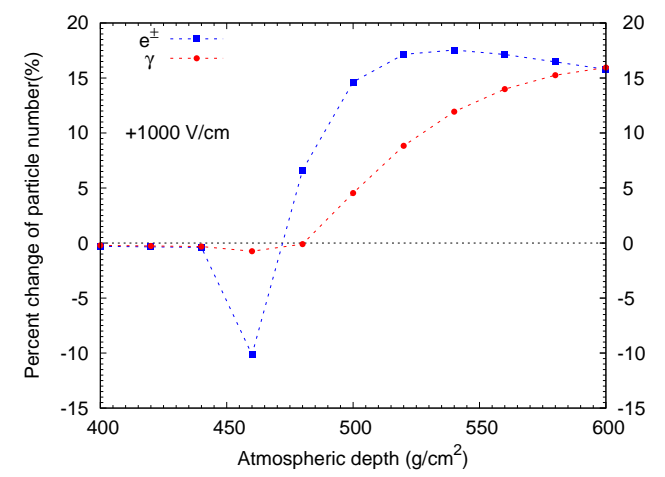

Fig. 4: Percent change of particles as a function of atmospheric depth in $1000 \mathrm{~V} / \mathrm{cm}$.

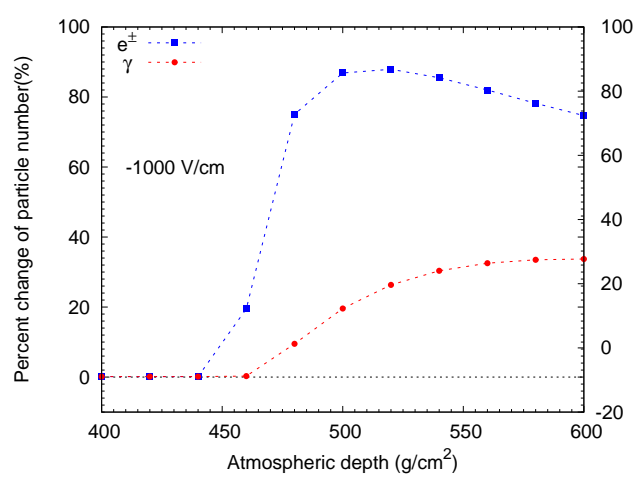

Fig. 5: Percent change of particles as a function of atmospheric depth in $-1000 \mathrm{~V} / \mathrm{cm}$.

\subsection{Percent change of particle number in different fields at Daocheng}

In order to get more information about the mechanism of secondary charged particles acceleration in thunderstorm electric field, we chose a series of electric field values from $-1000 \mathrm{~V} / \mathrm{cm}$ to 1000 V/cm. Fig. 6 and Fig. 7 show our simulating results. In a negative electric field (accelerating the electrons), the number of $e^{-}$increases, while the number of $e^{+}$reduces, and the total number of $e^{+}$and $e^{-}$increases with the increasing strength of electric field. When the field is positive (accelerating the positrons), the number of $e^{-}$reduces and the number of $e^{+}$increases. In the range $0-600 \mathrm{~V} / \mathrm{cm}$, the total number of $e^{+}$and $e^{-}$declines and the maximum decrease is $\sim 2.6 \%$. In the positive field greater than $600 \mathrm{~V} / \mathrm{cm}$, the sum of $e^{+}$and $e^{-}$increases with the increasing strength of the field. The percent change of photon number follows roughly the same pattern as the sum of $e^{+}$and $e^{-}$, except that the decrease happens in the $0-400 \mathrm{~V} / \mathrm{cm}$ range.

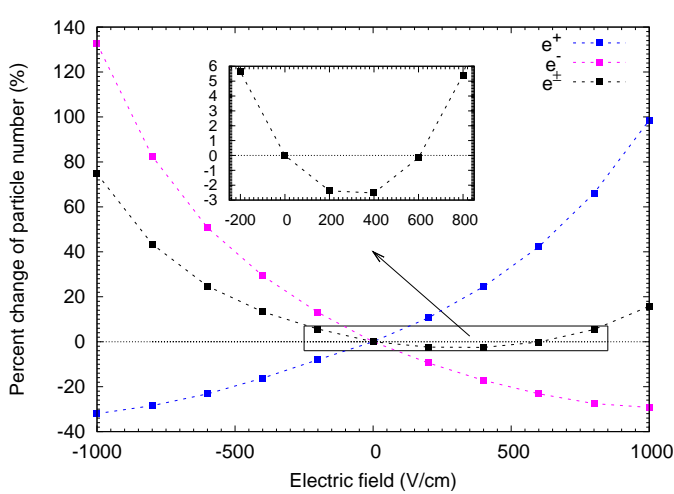

Fig. 6: Percent change of particle number as a function of electric field strength. (The illustration is the enlarged view of the field range from $-200 \mathrm{~V} / \mathrm{cm}$ to $800 \mathrm{~V} / \mathrm{cm})$.

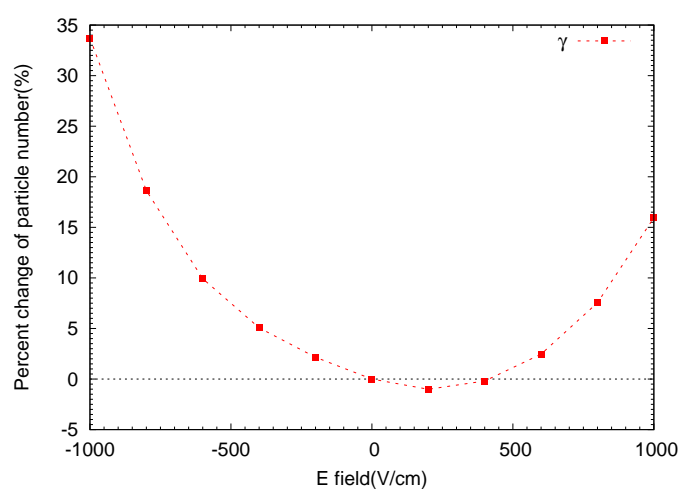

Fig. 7: Percent change of photo number as a function of electric field strength.

\section{Discussions}

As described above, the intensity variation amplitude highly depends on the strength and polarity of the electric field. Why does an electric field have a far greater impact on electrons than 
on positrons? And what causes the decline in the positive field? To better understand these results above, the energy distributions and the number ratio of $e^{-}$to $e^{+}$as a function of atmospheric are simulated. According to the Bethe theory [17], the slowing-down force due to collision of electron (or positron) with air varies with its energy, it decreases with increasing energy. The drag force reaches a minimum when the particle energy is $\sim 1.4 \mathrm{MeV}$, and then increases as the energy increases [18]. That means the thunderstorm field has more obvious effect on charged particle with smaller energy. Fig.8 shows energy distributions of $e^{+}$and $e^{-}$at Daocheng. In low energy regions, the proportion of electrons, which is the ratio of electrons in a certain energy region to the total number of electrons, is much larger than that of positrons. But the situation is reversed in higher energy regions. According to energy distribution, we obtained the average energy of electrons and that of positrons are $\sim 84 \mathrm{MeV}$ and $\sim 126 \mathrm{MeV}$, respectively. Besides, as illustrated in Fig.9, due to Compton scattering (making the number of electrons increase) and positron annihilation (resulting in a number decrease of positrons), the number of electrons is more than that of positrons in atmosphere. In addition, Compton Effect and positron annihilation effect increase with the increasing atmospheric depth. At the detect surface at Daocheng, the ratio of $N_{e}{ }^{-} / N_{e}+$ is $\sim 1.8\left(N_{e}{ }^{-}\right.$and $N_{e}{ }^{+}$ represent the number of electrons and the number of positrons, respectively). Based on the analysis above, the electron has a lower average energy and a larger number. It is easy to understand why the electric field has more significant effect on electrons than that on positrons, so does the intensity variations in an electric field with the same strength. I.e. in the strength of $1000 \mathrm{~V} / \mathrm{cm}$, the variation amplitude of positrons in positive field and electrons in negative field are $\sim 98.8 \%$ and $\sim 133.7 \%$, respectively. Though the number of electrons has a large increase in $-1000 \mathrm{~V} / \mathrm{cm}$, the particle multiplication does not experice a RREA process, but a normal accelerating one. The former will lead to an exponential increase in the number of electrons $[6,7]$.

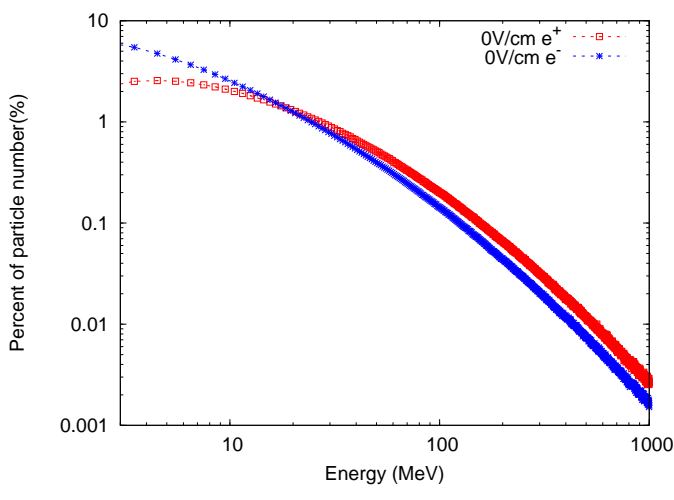

Fig. 8: Percent distributions of electrons and positrons as a function of energy.

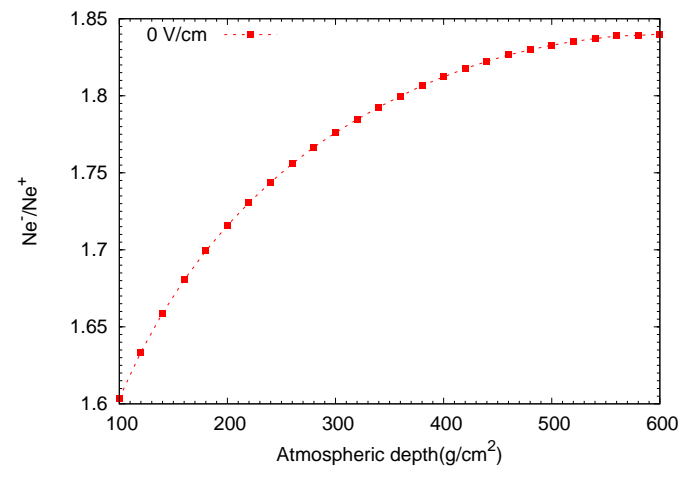

Fig. 9: Electrons to positrons ratio at $4400 \mathrm{~m}$ as a function of the atmospheric depth.

Due to electrons have a lower energy than positrons and are larger in number, the accelerating effects on electrons are more obviously than the decelerating effects on positrons in negative fields, resulting in the total number of $e^{+}$and $e^{-}$goes up with increasing electric field strength. For the same reason, the deceleration of electrons is more advantageous than the acceleration of positrons in a small positive field, the increase of positrons cannot compensate for the decrease of electrons. As a result, the total number of $e^{+}$and $e^{-}$will decrease in the field $0-600 \mathrm{~V} / \mathrm{cm}$. However, if the positive field strength becomes larger, the accelerating effects on positrons are dominant. The 
increase in positrons could compensate for the decrease of electrons and the total number starts to be increasing. This mechanism, explained in detail in [19], produces the asymmetric behavior shown in Fig. 6.

Though the electric field does not act on the photons directly, it still has significant effects on them. In $1000 \mathrm{~V} / \mathrm{cm}$, the total number of photos keeps increasing, at detect level the percentage increase is up to $\sim 15.9 \%$ in positive field and $\sim 33.7 \%$ in negative field. In generally, photos are produced in five main ways : positron annihilation, electron-positron annihilation, bremsstrahlung, Spontaneous radiation and Cherenkov radiation. In thunderstorm electric field, positive electric fields accelerate positrons to high energies, while negative electric fields accelerate electrons to high energies; the main generation mechanism of photons is bremsstrahlung. High-energy $e^{+}$or $e^{-}$may bremsstrahlung and deposit part of their energy into photons. Under certain conditions, photons will convert into $e^{+}$and $e^{-}$, which commonly called "pair-production". The process of repeated pair-production followed by bremsstrahlung is just like an electromagnetic cascade, causing the increases in the intensities of $e^{ \pm}$and $\gamma$ almost synchronously. The change of photon number follows roughly the same pattern as the sum of $e^{+}$and $e^{-}$, except that the decrease happens in the $0-400$ $\mathrm{V} / \mathrm{cm}$ range. This difference can be explained as follows. Owing to that the electric field does not directly act on the photons, the increase in the number of photons is just a secondary effect of the electric field accelerating the electrons or positrons. So, there is a little bit difference between them.

Taking the response of the detector into account, two points following are worthy of attentions. First, the number increases of secondary particles in electric field are mainly in a range of low energy. Otherwise, the detector is inefficient in responding to low energy particles. To sum up, we can expected that the intensity variation amplitude after the response of detector will become smaller than our simulations.

\section{Conclusions}

Monte Carlo simulations were performed to study the intensity changes of $e^{+}, e^{-}$and $\gamma$ in thunderstorms electric field at Daocheng. We found the intensity variation amplitude was closely related to the strength and polarity of the electric field.

In the strength of $1000 \mathrm{~V} / \mathrm{cm}$, the intensity variation amplitude of positrons in positive field and electrons in negative field are $\sim 98.8 \%$ and $\sim 133.7 \%$, respectively. And the number of photons is always increasing in any polarity fields, the increases of $\sim 15.9 \%$ and $\sim 37.8 \%$ correspond to $1000 \mathrm{~V} / \mathrm{cm}$ and $-1000 \mathrm{~V} / \mathrm{cm}$ respectively.

The total number of $e^{+}$and $e^{-}$increases with the strength of the field in the negative field or in the positive field greater than $600 \mathrm{~V} / \mathrm{cm}$, while a certain degree of decline $(\sim 2.6 \%)$ occurs in the positive field less than $600 \mathrm{~V} / \mathrm{cm}$. There are two main factors contributing to these simulating results. First, the number of positrons is less than that of electrons because of Compton scattering and positron annihilation. Secondly, the electric field has more obvious effects on electrons which have smaller energies and larger numbers.

Though the electric field does not act on the photons directly, it still has a significant effect on them. In thunderstorm electric field, the main generation mechanism of photons is the highenergy electrons or positrons releasing part of their energies by bremsstrahlung. The change of 
photon number follows roughly the same pattern as the sum of $e^{+}$and $e^{-}$, except that the decrease happens in the $0-400 \mathrm{~V} / \mathrm{cm}$ range.

Our simulations could be useful to analysis the forthcoming experimental data of LHAASO during thunderstorms and helpful to understand the acceleration mechanism of secondary charged particles caused by atmospheric electric field. The results also provide some clues for studying the correlations of the intensity variations between positrons, electrons and photons in the electric field.

\section{Acknowledgments}

This work is supported by the National Natural Science Foundation of China (NSFC) under the grant No. 11475141 and 11847307.

\section{References}

[1] D. R. MacGorman et al., The electrical nature of storms, Oxford Univ. Press, New York(1998)

[2] T.C. Marshall et al., Observed electric fields associated with lightning initiation, Geophys. Res. Lett. 32(2005)

[3] C. T. R. Wilson, The electric field of a thundercloud and some of its effects, Proc. Phys. Soc. 37 (1924)

[4] A.V. Gurevich et al., Runaway electron mechanism of air breakdown and preconditioning during a thunderstorm, Phys. Lett. A 165(1992)

[5] T.C. Marshall et al., Rocket and balloon observations of electric field in two thunderstorms, J. Geo- phys. Res. 100(1995)

[6] J.R. Dwyer, A fundamental limit on electric fields in air, Geophys. Res. Lett. 30(2003)

[7] E.M.D. Symbalisty et al., Finite volume solution of the relativistic Boltzmann equation for electron avalanche studies, IEEE Trans. Plasma Sci. 26(1998)

[8] G. J. Fishman et al., Discovery of intense gamma-ray flashes of atmospheric origin, Science 264(1994)

[9] D. M. Smith et al., Terrestrial gamma-ray flashes observed up to $20 \mathrm{MeV}$, Science, 307(2005)

[10] M. Marisaldi et al., Detection of Terrestrial Gamma-Ray Flashes up to 40-MeV by the AGILE Satellite, J. Geophys. Res.,115, A00E13(2010)

[11] V.V. Alexeenko et al., Transient variations of secondary cosmic rays due to atmospheric electric field and evidence for pre-lightning particle acceleration, Phys. Lett. A 301(2002)

[12] A. Chilingarian et al., Neutron bursts associated with thunderstorms, Phys. Rev. D 85(2012)

[13] J. F. Wang et al., Effect of thunderstorm electric field on intensity of cosmic ray muons, Acta Phys. Sin., 61(in Chinese)(2012)

[14] V.V. Alexeenko et al., Transient variations of secondary cosmic rays due to atmospheric electric field and evidence for pre-lightning particle acceleration, Phys. Lett. A 301(2002)

[15] B. Bartoli et al., Observation of the thunderstorm-related ground cosmic ray flux variations by ARGO-YBJ, Physical Review D, 97(2018)

[16] D. Heck et al., Report FZKA 6019, 1998, Forschungszentrum Karlsruhe, https://www.ikp.kit.edu/corsika/70.php

[17] H.A. Bethe, Theory of passage of swift corpuscular rays through matter, Ann. Phys. (N.Y) 397(1930)

[18] R. Roussel-Dupre et al., On runaway breakdown and upward propagating discharges, J. Geophys. Res.101(1996)

[19] X.X. Zhou et al., Effect of near-earth thunderstorms electric fi eld on the intensity of ground cosmic ray positrons/electrons in Tibet, Astropart. Phys. 84(2016) 